\title{
Use of multilocus sequence typing to infer genetic diversity and population structure of Lactobacillus plantarum isolates from different sources
}

Haiyan Xu, Wenjun Liu, Wenyi Zhang, Jie Yu, Yuqin Song, Bilige Menhe, Heping Zhang and Zhihong Sun ${ }^{*}$

\begin{abstract}
Background: Lactobacillus plantarum is a lactic acid bacterium $(L A B)$ of considerable industrial interest since it has an important role in the production of fermented food. In the present study, the genetic diversity and population structure within $186 \mathrm{~L}$. plantarum isolates was determined based on a novel MLST scheme employing eight housekeeping genes. These isolates had originated from different sources and geographic regions: 179 isolates were from our own culture collection and originated from China and Mongolia and seven isolates were type or reference isolates from other collections.

Results: The results showed that 179 isolates and seven reference isolates could be assigned to 73 different sequence types (STs), forming ten clonal complexes (CCs) and 23 singletons. There were 158 polymorphic sites detected in total, and the nucleotide diversity per site varied from 0.00401 in clpX to 0.03220 in groEL. The minimum spanning tree analyses suggested that the evolution of $L$. plantarum isolates have little relationship with ecological sources have similar nucleotide diversity. Phylogenetic trees and structure indicated that there were six lineages in the L. plantarum isolates used in our study. Split-decomposition and ClonalFrame analysis indicated that recombination had occurred throughout the population of L. plantarum, but it occurred at a low frequency in these eight loci.
\end{abstract}

Conclusion: We deduced that L. plantarum isolates from the same ecological niches have similar genetic diversity and population structure. The MLST scheme presented in this study provides abundant sequence data for L. plantarum and enabled global comparisons of isolates associated with various environmental origins to be made. This will further advance our understanding of the microbial ecology of this industrially important LAB.

Keywords: Lactobacillus plantarum, Multilocus sequence typing, Housekeeping genes, Genetic diversity, Population structure

\section{Background}

Lactobacillus plantarum, is an ubiquitous species of lactic acid bacterium (LAB) that is found in a wide range of different ecological niches including vegetable, meat and dairy substrates, and the gastro-intestinal tract [1]. The diversity of niches occupied by L. plantarum is related to its capacity to ferment a broad range of sugars [2].

\footnotetext{
*Correspondence: sunzhihong78@163.com
Key Laboratory of Dairy Biotechnology and Engineering, Ministry of
Education, Inner Mongolia Agricultural University, Hohhot, Inner Mong

* Correspondence: sunzhihong78@163.com
Key Laboratory of Dairy Biotechnology and Engineering, Ministry of
Education, Inner Mongolia Agricultural University, Hohhot, Inner Mo

* Correspondence: sunzhihong78@163.com
Key Laboratory of Dairy Biotechnology and Engineering, Ministry of
Education, Inner Mongolia Agricultural University, Hohhot, Inner Mongolia 010018, China
}

\section{Biomed Central}

(c) 2015 Xu et al. Open Access This article is distributed under the terms of the Creative Commons Attribution 4.0 International License (http://creativecommons.org/licenses/by/4.0/), which permits unrestricted use, distribution, and reproduction in any medium, provided you give appropriate credit to the original author(s) and the source, provide a link to the Creative Commons license, and indicate if changes were made. The Creative Commons Public Domain Dedication waiver (http://creativecommons.org/publicdomain/zero/1.0/) applies to the data made available in this article, unless otherwise stated.

Isolates of $L$. plantarum have been widely used as starter cultures in the production of fermented vegetables and other crops [3-5], fermented dairy products such as cheese $[6,7]$, fermented meat products $[8,9]$, and as a probiotic for humans and animals [10].

Lactobacillus plantarum is closely related to the species Lactobacillus fabifermentans, Lactobacillus paraplantarum, Lactobacillus pentosus and Lactobacillus xiangfangensis and, within L. plantarum, a number of subspecies have been identified. These include L. plantarum subsp. argentoratensis and L. plantarum subsp. 
plantarum [11]. These closely related species and subspecies have similar nutritional requirements and, as such, they are difficult to separate using classical methods [12]. However, interspecific and intraspecific differentiation is an important preliminary step in the selection of starter cultures. Technological, probiotic, antimicrobial and sensorial attributes are strain-specific and so it is important to be able to distinguish between isolates with different properties [13]. For this reason, a great number of, molecular, techniques have been developed for the identification and typing of $L$. plantarum isolates. These include randomly amplified polymorphic DNA, pulsed-field gel electrophoresis, amplified fragment length polymorphism, repetitive element PCR and multilocus sequence typing (MLST) [13-17].

The MLST method was first described in 1998 [18] and since then has been used widely to study the population structure of important bacterial pathogens $[19,20]$. Recently, research evidence has suggested that it could also be a powerful technique for typing $\mathrm{LAB}$, allowing precise identification and easy comparison/exchange of results between different laboratories [21-23]. Previously, MLST has been used for the typing of L. plantarum isolates from fermented fruits and vegetables [16], and from silage, wine, pickled cabbage and cheese [13]. However, the number of isolates evaluated was rather limited, making any interpretation of the genetic heterogeneity of L. plantarum very difficult, and, potentially misleading. In the present study, a new MLST scheme was established and applied to a large number of L. plantarum isolates (179 plus seven reference and type isolates) from a wide range of geographic regions and different sources, to encompass the full genetic diversity and population structure of the species.

\section{Result}

\section{Sequence types and clonal complexes}

The partial sequences of the eight housekeeping genes (from 179 isolates and seven reference isolates of $L$. plantarum) ranged in size from 415 to $641 \mathrm{bp}$. A total of 73 unique STs were resolved, of which 44 corresponded to single isolates (Additional file 1: Table S1). ST47 contained the largest number of isolates, 19, which represented $10.2 \%$ of all isolates evaluated (Additional file 1: Table S1). Furthermore, four of the reference isolates were assigned to STs (and CCs) with other isolates tested while the remaining three reference isolates were singletons in their own ST (Additional file 1: Table S1).

The 73 STs could be classified into ten CCs and 23 singletons using eBURST (Additional file 1: Table S1). The ten CCs contained $77.5 \%$ of the isolates (Additional file 2: Figure S1). CC1 (22 STs) contained 70 isolates, ST14 was associated with seven single locus variants (SLVs), which was the predicted founder genotype
[24]. CC2, CC3 and CC4 contained six (11 isolates), five (27 isolates) and five (15 isolates) STs, respectively. All other CCs contained fewer than five STs, each with a limited number of isolates. The links among CCs and singletons disclosed by eBURST profile were consistent with minimum spanning trees (Additional file 2: Figure S1 and Fig. 1).

\section{Genetic diversity}

The nucleotide diversity within each of the eight housekeeping genes for all isolates was calculated and between seven ( $p y r G$ ) and 15 (murE) alleles were found (Table 1). In total there were 158 polymorphic sites with between eight and 63 per gene (Table 1). The nucleotide diversity per site ( $\pi$, the average number of nucleotide differences per site between two randomly-selected isolates) among the eight genes varied from $0.00401(\operatorname{clpX})$ and 0.03220 (groEL). In addition, the number of sSNPs (synonymous single nucleotide polymorphism sites) was greater than the number of nSNPs (non-synonymous Single Nucleotide Polymorphism sites) except for groEL, which had 59 nSNPs and 6 sSNPs, the largest among all the genes. The DNA G + C\% content of the different gene fragments ranged from 43.10 (uvrC) to $51.38 \%$ (murE) and the proportion of concatenated sequences was $45.28 \%$; The $d_{N} / d_{S}$ ratios for the genes were between $0($ rec $A)$ and 0.2692 (murC) respectively, and were all lower than 1 (Table 1).

\section{Phylogenetic relationships analysis}

Analysis of the allelic profiles of all 186 isolates generated a minimum spanning tree that provided an intuitive view of the phylogenetic relationships between STs, singletons, CCs and lineages (Fig. 1). One dominant group (in blue shading) and some small clades (Clades I-V) were discerned. The dominant group included $\mathrm{CC} 1$, CC6, CC7, CC9 and some singletons, and primarily contained isolates from Sichuan pickle (to the left of the group in Fig. 1) or sourdough and a small number of isolates were from fermented dairy foods (to the right of the group in Fig. 1). Clade I contained seven isolates from four STs (ST38, ST54, ST64 and ST67) of which six isolates came from pickles. Clade II contained isolates with the greatest diversity of ecological sources, although isolates in ST3 all originated from fermented dairy foods including reference isolate CGMCC6312 [25]. Clade III was composed of five STs, each from a different region, but all containing isolates from fermented dairy foods. Similarly, isolates in Clade IV were all from fermented dairy foods. Most of the STs in Clade V were isolated from pickles. 


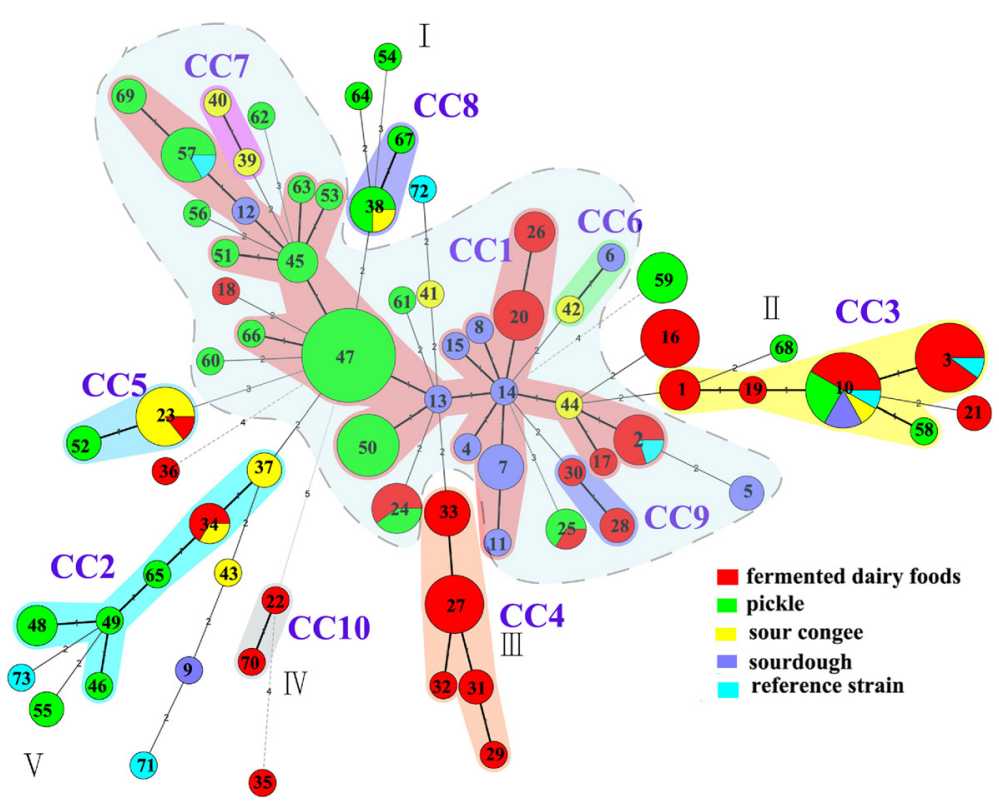

Fig. 1 Minimum spanning trees analysis of the $186 \mathrm{~L}$. plantarum isolates evaluated based on eight loci. Each circle corresponds to a sequence type (ST), and the circle size corresponds to the number of isolates sharing the same ST, the circle was coded by assigning the same colour to identical ecological source. The shaded zones between certain groups of circles indicate that these profiles belong to the same CC. Numerals connecting the circles indicate the number of allelic differences between the profiles. The strength of the link (bold, plain, or discontinuous) is correlated to the genetic similarity (number of common alleles) between profiles. Black lines connecting pairs of STs indicate that they share seven (thick lines), six (thin) or five alleles (dotted). No lines are present between pairs of STs that share less than four alleles

\section{Population structure}

The concatenated sequences size of the eight housekeeping genes of $186 \mathrm{~L}$. plantarum isolates was $4251 \mathrm{bp}$. A Neighbour-joining tree of the concatenated sequences of 73 STs was in almost complete agreement with the STRUCTURE which revealed six major ancestral populations, or clusters (Cluster 1-6) (Fig. 2). From the Neighbour-joining tree Cluster 1 (blue) comprised 25 STs and 59 isolates, of which 94.9 \% came from nondairy sources, and included reference isolates CGMCC0847 and CGMCC12167 and the type isolate ATCC $14917^{\mathrm{T}}$. Cluster 2 (red) contained eight STs, and the distances amongst ST22, ST35 and ST70 from dairy sources were closer than amongst the other STs from non-dairy sources. The $20 \mathrm{STs}$ in Cluster 3 (bottle green) included isolates from multiple sources, although $62.0 \%$ of 50 isolates were from fermented dairy foods from different areas, including reference isolate NCIMB8826. Cluster 4 (orange) contained 44 isolates in 11 STs; $77.3 \%$ of these isolates were from fermented dairy food and included

Table 1 Descriptive analysis of MLST data genetic variability at L. plantarum loci

\begin{tabular}{|c|c|c|c|c|c|c|c|c|}
\hline \multirow[t]{2}{*}{ Gene } & \multirow[b]{2}{*}{ Length (bp) } & \multicolumn{2}{|l|}{ No. of } & \multirow[t]{2}{*}{$\pi^{\mathrm{a}} /$ site } & \multirow{2}{*}{$\begin{array}{l}\mathrm{G}+\mathrm{C} \\
\text { content } \\
\text { (mol\%) }\end{array}$} & \multirow[b]{2}{*}{ SSNP } & \multirow[b]{2}{*}{$n S N P$} & \multirow[t]{2}{*}{$d_{N} / d_{S}^{b}$} \\
\hline & & Alleles & Polymorphic sites & & & & & \\
\hline$c l p X$ & 443 & 9 & 8 & 0.00401 & $45.77 \%$ & 7 & 1 & 0.0429 \\
\hline groEL & 641 & 11 & 63 & 0.03220 & $43.93 \%$ & 6 & 59 & 0.0177 \\
\hline murC & 462 & 11 & 10 & 0.00502 & $43.66 \%$ & 5 & 4 & 0.2692 \\
\hline murE & 607 & 15 & 22 & 0.00855 & $51.38 \%$ & 16 & 6 & 0.0436 \\
\hline phes & 536 & 12 & 15 & 0.00693 & $46.09 \%$ & 10 & 5 & 0.0648 \\
\hline pyrG & 415 & 7 & 9 & 0.00803 & $43.28 \%$ & 8 & 1 & 0.0255 \\
\hline recA & 546 & 9 & 10 & 0.00549 & $45.00 \%$ & 10 & 0 & 0 \\
\hline uvrC & 620 & 12 & 21 & 0.01166 & $43.10 \%$ & 18 & 3 & 0.0279 \\
\hline concatenated & 4251 & 80 & 158 & 0.00464 & $45.28 \%$ & 80 & 79 & 0.0401 \\
\hline
\end{tabular}

${ }^{a}$ Mean pairwise nucleotide difference per site

${ }^{\mathrm{b}} d_{\mathrm{N}} / d_{\mathrm{S}}$ represents the ratio of nonsynonymous to synonymous substitutions 


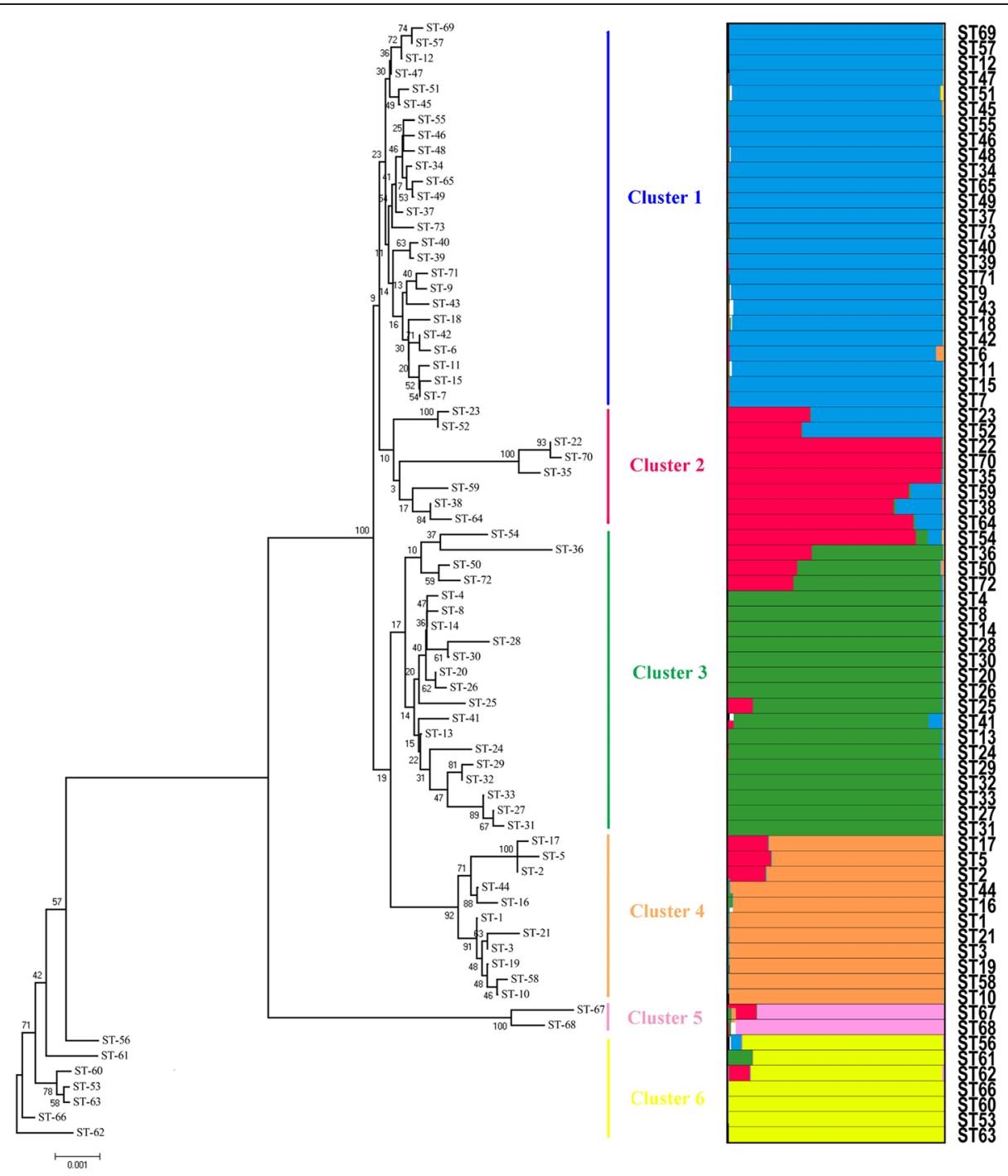

Fig. 2 Neighbour-Joining tree and Ancestry of 73 L. plantarum STs. The left of the figure: Neighbour-Joining tree constructed from 73 concatenated nucleotide sequences of eight genes used in MLST. Bootstrap values are indicated for all branches. The right of the figure: The sources of ancestry of each unique ST from six ancestral populations by STRUCTURE. Each ST is represented by a single line with the ST designation at the top consisting of colour stacked bars that indicate the proportion of ancestry from each of six populations (blue, red, bottle green, orange, pink and yellow)

reference isolates CGMCC12986 and CGMCC6312. Cluster 5 (pink) contained only two STs, both with isolates from pickle. Cluster 6 (yellow) contained seven STs, all with isolates from pickles.

The STRUCTURE showed six distinct clusters representing six ancestral populations (shown in six different colours) within 73 STs (each vertical line represented one ST). Isolates in Cluster 1 were isolated from nonfermented dairy products from different regions. However, some isolates from the same food origin were also divided between Clusters; isolates from pickle in Sichuan were divided between Clusters 5 and 6. Cluster 2, 3 and 4 were very diverse in origin but we identified the same ancestral sources with particular. The ancestry of each isolate was estimated as the summed probability of derivation from each cluster over all polymorphic nucleotides (shown at the right of Fig. 2). There were little admixture of ancestral sources in these clusters, with the exception of ST2, ST5, ST17, ST23, ST36, ST38, ST50, ST52 and ST72 (a percentage of assignment to a cluster lower than $85 \%$ is considered as threshold for admixture), which seemed to have acted frequently both as donors and as recipients of recombination exchanges.

\section{Recombination analysis}

Analysis of all isolates indicated the index of association $\left(I_{\mathrm{A}}\right)$ of $-0.0382(P=0.66)$ and standardized index of association $\left(I_{\mathrm{A}}^{\mathrm{S}}\right)$ of $-0.0055(P=0.57)$, suggesting no significant linkage disequilibrium and indicating a tendency for free recombination between the alleles. 


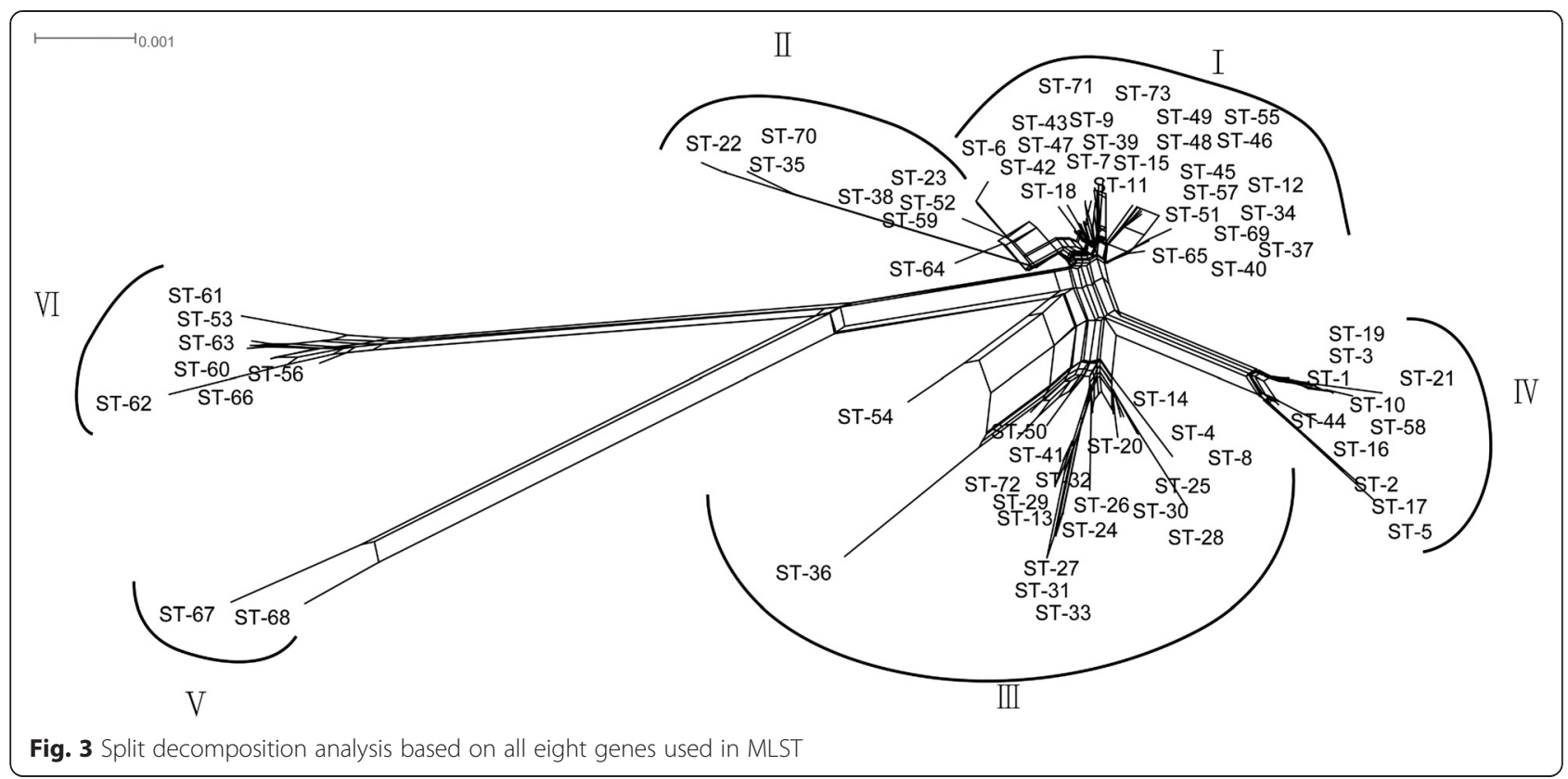

Split decomposition analysis of the concatenated $\operatorname{clp} X$, groEL, murC, murE, phesS, pyrG, recA and $u v r C$ gene sequence fragments displayed in a split decomposition tree (Fig. 3) showed a network-like structure with multiple parallel paths. There were six distinct clusters in the split decomposition tree, which was consistent with the phylogeny observed previously in the neighbour-joining tree and from STRUCTURE analysis.

ClonalFrame analysis estimated that recombination happened less frequently than mutation $(\rho / \theta=0.18 ; \mathrm{Cl}$ 95: [95\% credibility interval]: $0.07-0.33$ ) and was less effective in introducing polymorphisms $(\gamma / \mathrm{m}=0.64 ; \mathrm{Cl}$ 95: 0.31-1.03). Clonal genealogy inferred from analysis of the data by ClonalFrame showed six clusters (Fig. 4). Clusters 4,5 and 6 were the same clusters as identified by STRUCTURE (Fig. 2). The remaining three clusters found by STRUCTURE contain the remaining isolates in Fig. 3, but do not represent the different clusters in Fig. 4, Clusters 1, 2 and 3 from STRUCTURE analysis, were divided into smaller groups by ClonalFrame.

\section{Discussion}

Lactobacillus plantarum is a LAB of considerable industrial interest, which plays a key role in the production of fermented food. Nevertheless the genetic diversity and population structure of this microorganism from different ecological origins had not been fully explored until this study. Here we used a new MLST scheme to examine isolates from a diversity of ecological sources. The new MLST scheme relied on sequencing of 400-650 bp fragments from eight housekeeping genes, which was a method previously proved to be useful for elucidating the phylogenetic relationships and evolution of fungal isolates [26]. However, an independent MLST scheme had been described [13] in which 16 isolates were tested for seven housekeeping gene fragments. Compared to the MLST schemes, an average of ten distinguishable alleles per locus among the 73 STs were identified, which is more than the average of 5 alleles observed from 16

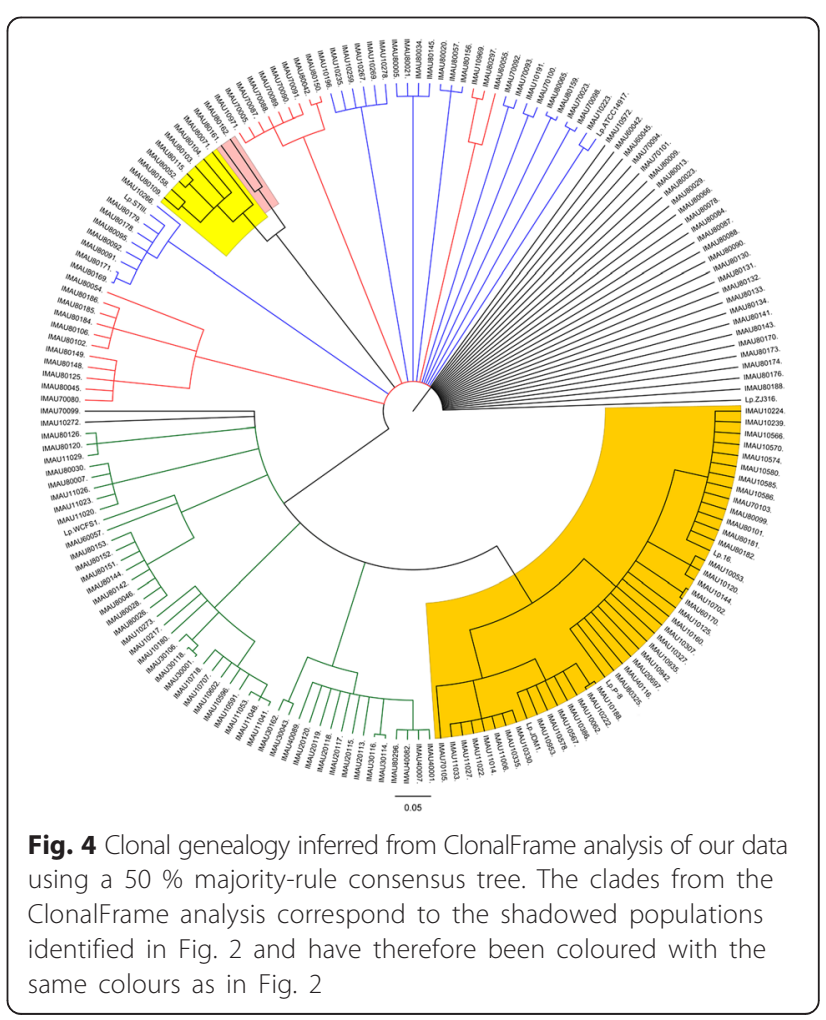


Lactobacillus plantarum isolates. Our MLST scheme has stronger typing discrimination for Lactobacillus plantarum.

By comparison, the $\pi$ values obtained for L. plantarum isolates in our study were higher than $L$. delbrueckii ranged from 0.0051 to 0.0096 [27] and for L. plantarum ranged from 0.0004 to 0.0072 [13], indicating a higher degree of intragenic nucleotide polymorphism in our isolates [13]. The sequences of eight housekeeping genes examined by MLST had mol\% G + C contents that were similar to the genome of L. plantarum ATCC $14917^{\mathrm{T}}$ [28]. This suggests that these genes had been present in L. plantarum for a long period of time and were relatively conserved. Overall the $d_{N} / d_{S}$ ratios of the selected genes were lower than 1 , which is typical of housekeeping genes and desirable in MLST schemes [29].

We investigated the evolutionary relationships amongst our isolates of L. plantarum by eBURST, based on alleles, and constructed a minimal spanning tree (Additional file 2: Figure S1 and Fig. 1). According to the minimal spanning tree, the dominant groups of isolates in blue shading and Clade I and Clade V, were associated with plant origins with most isolates coming from pickles, sourdough and sour congee. Notably in this dominant group, the reference isolate CGMCC0847 from kimchi [30] belonged to ST57 with other isolates from pickle; likewise, reference isolate CGMCC12986, a commercial lactic acid bacterium [31], was assigned to ST2 with other isolates from fermented dairy foods. Isolates in Clades III and IV, were all isolated from dairy sources, suggesting that L. plantarum isolates from identical or similar environmental sources were closely related. Curiously, the type isolate of L. plantarum, ATCC $14917^{\mathrm{T}}$ from pickled cabbage shared six alleles with ST9 which was from sourdough [1], so the relationship between them was also close. Similarly, the reference isolate CGMCC12167 from a faecal sample was a two loci variant of ST49 isolated from pickle (Fig. 1). Furthermore, the reference isolates CGMCC12986 and CGMCC6312 were assigned to the same STs, with other isolates, and were isolated from identical sources. Therefore we can conclude that isolates from the same environmental sources have similar nucleotide diversity, maybe it's just because different kinds of home-made fermented foods have an effect on the genetic evolution of L. plantarum, which existed in these traditional foods for a long time based on the special production process of these home-made foods. Our findings were different from de las Rivas, where there was little relationship with the sources of the isolates [13]. De las Rivas described most of the types were represented by a single strain, only strains isolated from the same source, such as strains CECT 223 and 224, and strains RM71 and 72 shared the same ST, but our results corroborated $39.7 \%$ of the isolates shared STs. In addition, the type isolate of L. plantarum ATCC $14917^{\mathrm{T}}$ and reference isolate
NCIMB8826 represented a single ST in both schemes, respectively, maybe because our isolates were isolated from some special fermented foods. Similarly, the report on $L$. sanfranciscensis confirmed manufacturing environment was the predominant factor influencing the dominance of an isolate on the evolution of lactobacilli population [32]. Cai also proved that $L$. casei was adaptive evolution towards specific niches [33].

Results from the Neighbour-Joining tree corresponded well to the results from STRUCTURE analysis with respect to the identification of Clusters. STRUCTURE analysis recognized six ancestral sources of polymorphism within the L. plantarum isolates evaluated and these six groups had relationships with different environmental source. In addition, most STs were single ancestral source to contribute more than $85 \%$. However, within the entire dataset, some STs (16.1\% of all isolates) contained significant ancestry from multiple sources, where extensive recombination has yielded a highly heterogeneous set of isolates with multiple sources of ancestry (Fig. 2). This confirms previous studies demonstrating that the recombination plays a role in creating genetic heterogeneity in L. plantarum [13].

It is now clear that recombination plays a driving role in the evolution of bacteria [34], but little was known about the details of the recombination process within L. plantarum. In our study, multilocus linkage disequilibrium was analyzed using $I_{A}$ and $I_{A} \mathrm{~S}$ for the recombination measurement of L. plantarum. The values of $I_{A}$ and $I_{A}{ }^{\mathrm{S}}$ were close to zero, which confirmed the importance of recombination in the distribution of alleles. Consistently, the split decomposition analysis of concatenated sequences displayed a very complex interconnecting network structure. Therefore, our data suggest that recombination has contributed to the evolution of L. plantarum, further confirmed by limited and frequent gene flow across clusters. These events may have facilitated rapid adaptation of $L$. plantarum to different environments. This is in agreement with previous reports that recombination is an important source of diversity in LAB [16].

Likewise, the ClonalFrame analysis confirmed that homologous recombination contributed to the evolution of $L$. plantarum to some degree. Based on the combined evidence from STRUCTURE and ClonalFrame analyses, Cluster 4, 5 and 6 can confidently be called lineages of $L$. plantarum. Two of these three lineages (Cluster 5 and 6) were closely related and they were all isolated from pickle. Interestingly, some clades in the ClonalFrame analysis were defined as groups of isolates that mainly had the same ancestry as in the Structure analysis, indicating that recombination happened predominantly between related isolates during the evolution of L. plantarum [34, 35]. Moreover, it remains likely that other small clades and single isolates exist and might be identified if a larger number of isolates were evaluated. 


\section{Methods}

Isolates, media and culture conditions

Isolates evaluated were all from the Lactic Acid Bacteria Culture Collection of the Inner Mongolia Agriculture University, China (IMAU) with the exception of the reference and type isolates that were from the American Type Culture Collection (ATCC: isolate ATCC14917 ${ }^{\mathrm{T}}$ ), the China General Microbiological Culture Collection Center (CGMCC: isolates CGMCC 0847, 6312, 12167 and 12986), and the National Collection of Industrial and Marine Bacteria (NCIMB: isolates NCIMB 8826 and 41875) (Additional file 1: Table S2, Table S3). The IMAU isolates were originally isolated from home-made pickle, sour congee, sourdough and fermented dairy foods (cows' milk, goats' milk and mares' milk) sampled in the Sichuan, Qinghai and Xinjiang provinces of China, the Inner Mongolia and Tibet autonomous regions of China, and the Dornogovi province of Mongolia. 187 L. plantarum strains were isolated from 187 different fermented food samples, which had been in mature stage. These home-made fermented foods are manufactured by natural fermentation with natural starter cultures (a stable microorganism formed during fermented process) from old fermented food, without the use of any commercial starter cultures (isolated and cultured by companies or departments to sell). In this way, L. plantarum was kept and handed down through the generations along with traditional home-made food recipes. All L. plantarum isolates had been achieved by $16 \mathrm{~S}$ rRNA sequences [36-42] (Additional file 1: Table S2). Reference isolates were from dairy products, pickles, saliva and faecal samples from China, USA and UK (Additional file 1: Table S3). Each isolate was grown at $37{ }^{\circ} \mathrm{C}$ over night in $5.0 \mathrm{ml}$ de Man Rogosa Sharpe broth (MRS; OXOID, CM0359B, Germany).

\section{DNA extraction and PCR amplification}

Genomic DNA was extracted from each isolate using the methods of $\mathrm{Yu}$ [40]. DNA extraction the cells were pelleted by centrifugation at $8000 \times \mathrm{g}$ and resuspended in TE. Briefly, $1 \mathrm{~mL}$ of sample was frozen with liquid $\mathrm{N}_{2}$ for $5 \mathrm{~min}$ and thawed in a water bath at $65{ }^{\circ} \mathrm{C}$. Then $600 \mu \mathrm{L}$ of $400 \mathrm{mmol} \mathrm{L}^{-1} \mathrm{NaOH}$ and $300 \mu \mathrm{L}$ of $400 \mathrm{~g} \mathrm{~L}^{-1}$ trisodium citrate dihydrate were added. The mixed sample was centrifuged at $8000 \times \mathrm{g}$ for $5 \mathrm{~min}$. The supernatant fluid was discarded. Afterwards, the sediment was dissolved in sterile ultrapure water. Then $50 \mu \mathrm{L}$ of $100 \mathrm{~g} \mathrm{~L}^{-1}$ sodium dodecyl sulfate and $10 \mu \mathrm{L}$ of $10 \mathrm{mg} \mathrm{mL}^{-1}$ proteinase $\mathrm{K}$ solution were added and the mixture was incubated at $55^{\circ} \mathrm{C}$ for $1 \mathrm{~h}$. After incubation, $10 \mu \mathrm{L}$ of $5 \mathrm{~mol} \mathrm{~L}^{-1} \mathrm{NaCl}$ and $100 \mu \mathrm{L}$ of cetyltriethyl ammnonium bromide/ $\mathrm{NaCl}(1: 9 \mathrm{w} / \mathrm{w})$ were added and the mixture was further incubated at $65{ }^{\circ} \mathrm{C}$ for $10 \mathrm{~min}$. Subsequently, the mixture was extracted with 1 volume of phenol/chloroform/isoamyl alcohol (25:24:1 v/v/v) and chloroform/ isoamyl alcohol $(24: 1 \mathrm{v} / \mathrm{v})$ twice. Finally, DNA was precipitated by adding 0.1 volume of $3 \mathrm{~mol} \mathrm{~L}^{-1}$ sodium acetate to the water phase followed by 1 volume of ice-cold isopropyl alcohol. The DNA was collected, washed and dissolved in $50 \mu \mathrm{L}$ of sterile ultrapure water. Purified DNA was diluted to a final concentration of $100 \mathrm{ng} / \mu \mathrm{L}$ for evaluation. For each isolate, genomic DNA was used as a template for PCR amplification of MLST loci using an automatic thermal cycler (PTC-200, MJ Research, Waltham, MA). Thermal cycling conditions were: $94{ }^{\circ} \mathrm{C}$ for $5 \mathrm{~min}$; 30 cycles of $94{ }^{\circ} \mathrm{C}$ for $1 \mathrm{~min}$, the appropriate temperature for the gene of interest for $1 \mathrm{~min}$ (Table 2), and $72{ }^{\circ} \mathrm{C}$ for $2 \mathrm{~min}$; a final elongation step of $72{ }^{\circ} \mathrm{C}$ for $10 \mathrm{~min}$. Each PCR reaction was performed in a volume of $50 \mu \mathrm{L}$ containing $150 \mathrm{ng}$ of genomic DNA, $8 \mathrm{mM}$ of each dNTP, 10 pmol of each primer and $2.5 \mathrm{U}$ Taq polymerase in $1 \times$ PCR buffer (with $\left.\mathrm{Mg}^{2+}\right)$. PCR products were electrophoresed in a $1.2 \%$ agarose gel. The PCR products were sequenced by the Shanghai Majorbio Bio-pharm Technology Corporation. The same primers were used for PCR and sequencing of both DNA strands.

\section{MLST gene selection}

Genes were selected on the basis of their location on the chromosome (preferably evenly separated across the entire genome), the functions of the encoded proteins (preferably conserved and well characterized) and their presence in all isolates as a single copy [43]. Eight housekeeping genes (clpX, groEL, murC, murE, pheS, pyrG, recA and $u v r C$ ) were selected for MLST analysis of L. plantarum isolates. These housekeeping genes were conserved sequence, their wide distribution across the chromosome, and their mutually unlinked in location. The $c l p X$ encodes ATP-dependent protease ATP-binding subunit ClpX, groEL encodes chaperonin GroEL, murC encodes UDP-N-acetylmuramyl tripeptide synthase, $m u r E$ encodes UDP-Nacetylmuramoylalanyl-D-glutamate-2, 6diaminopimelate ligase, pheS encodes phenylalanyl-tRNA synthase alpha subunit, pyrG encodes CTP synthetase, $r e c A$ encodes recombinase $\mathrm{A}, u v r C$ encodes excinuclease $\mathrm{ABC}$ subunit $\mathrm{C}$. The genes pyrG, groEL and rec $A$ had been used in a previous study on Lactobacillus delbrueckii [27], and pheS in a study on Lactococcus lactis [44]. The genes clpX, murC, murE and uvrC were known to have single nucleotide polymorphisms (SNPs) in one of the reference L. plantarum isolates, CGMCC6312 (Genbank number: CP005942). Primers for these genes were designed using the Primer Premier 5.0 programme (Premier Biosoft International) based on the complete genome of L. plantarum isolate CGMCC6312 (Table 2). For subsequent comparison we downloaded from Genbank the sequences of these eight genes in the reference and type isolates of L. plantarum that we used in this study (Additional file 1: Table S3). 
Table 2 The information of primers and housekeeping genes for MLST of L. plantarum

\begin{tabular}{|c|c|c|c|c|c|}
\hline Gene & Template Length (bp) & Gene function & PCR primer & Sequences $\left(5^{\prime}-3^{\prime}\right)$ & Annealing Temperature \\
\hline \multirow[t]{2}{*}{ phes } & 717 & Phenylalanyl-tRNA synthase alpha subunit & phes_primerF & CCGTGAAGAACTGGAACA & $49^{\circ} \mathrm{C}$ \\
\hline & & & phes_primerR & CCTAACCCAAAGGCAAAA & \\
\hline \multirow[t]{2}{*}{ pyrG } & 668 & CTP synthase & pyrG_primerF & AGTGATTTAGGTTCCGACAA & $52^{\circ} \mathrm{C}$ \\
\hline & & & pyrG_primerR & TGCATTCCCAAGCAGATA & \\
\hline \multirow[t]{2}{*}{ uvrC } & 763 & UvrABC system protein $C$ & uvrC_primerF & GATCATTTATGTGGGTAAGGC & $53^{\circ} \mathrm{C}$ \\
\hline & & & uvrC_primerR & TGACACTACTGGGAACAAGC & \\
\hline \multirow[t]{2}{*}{ recA } & 689 & Recombinase A & recA_primerF & TITAGTTGTTGACTCGGTGGC & $58^{\circ} \mathrm{C}$ \\
\hline & & & recA_primerR & TTCCGCTGGTGTCGCTTT & \\
\hline \multirow[t]{2}{*}{$c l p X$} & 563 & ATP-dependent Clp protease subunit X & clpX_primerF & ATCGCCAAGAAGAGTGAA & $52^{\circ} \mathrm{C}$ \\
\hline & & & clpX_primerR & ATAATCGAGCGTAGACCC & \\
\hline \multirow[t]{2}{*}{ murC } & 641 & UDP-N-acetyl muramate-alanine ligase & murC_primerF & TATCGCTCCCACCAGTTA & $51^{\circ} \mathrm{C}$ \\
\hline & & & murC_primerR & CGGCCAAGATTTCCTTAT & \\
\hline \multirow[t]{2}{*}{ groEL } & 737 & 60 kDa chaperonin & groEL_primerF & CGGCTACTTATCACAATACA & $58^{\circ} \mathrm{C}$ \\
\hline & & & groEL_primerR & GCCTTCTAAACCAGCATT & \\
\hline \multirow[t]{2}{*}{ murE } & 750 & UDP-N-acetylmuramyl tripeptide synthase & murE_primerF & ACTAATAAGGTCGCTGTTCTG & $55^{\circ} \mathrm{C}$ \\
\hline & & & murE_primerR & TTAAGCGGTTCTTCACT & \\
\hline
\end{tabular}

\section{MLST data analysis}

Sequence alignment, trim, analysis and the identification of polymorphic sites were performed using the MEGA 6.0 software package [45]. BioNumerics Software (version 6.0, Applied-Maths, SintMaartens-Latem, Belgium) was used for sequence assembly, definition of alleles and the construction of a minimum spanning tree. For each of the eight genes used in MLST, unique nucleotide sequences defined an allele. Unique allelic profiles for isolates consisted of unique combination of alleles (one from each gene), and were used to unambiguously define the sequence type (ST) of that isolate; several isolates could belong to the same ST if they shared the same allelic profile. Groups of isolates with closely related STs were grouped within clonal complexes(CCs) using eBURST [46] (http://eburst.mlst.net/).

The START version 2.0 programme [47] was used to calculate the number of polymorphic sites, the mean $G$ $+\mathrm{C} \%$ content of the DNA and the $d_{N} / d_{S}$ ratios (where $d_{S}$ is the number of synonymous substitutions per synonymous site, and $d_{N}$ is the number of non-synonymous substitutions per non-synonymous site). Using this software we also evaluated intragenic recombination within the eight MLST genes. Multilocus linkage disequilibrium between alleles was measured using the index of association $\left(I_{\mathrm{A}}\right)$ and standardized index of association $\left(I_{\mathrm{A}}^{\mathrm{S}}\right)$ [48]. These statistics are expected to be zero when the alleles are in linkage equilibrium, which also indicates that frequent recombination events exist. We used DnaSP version 5.0 to calculate the nucleotide diversity ( $\pi)$ per site and polymorphic sites [49].
The sequences of the eight housekeeping genes of 186 L. plantarum isolates were concatenated. Phylogenetic trees based on concatenated sequences were constructed using the Neighbour-Joining method, with a Kimura two-parameter distance model in the MEGA 6.0 software package. The Bayesian analysis software STRUCTURE with an associated linkage model was used to identify the ancestral populations of each isolate [50]. We use a Bayesian clustering approach to assume a model in which there are $K$ populations, each of which is characterized by a set of allele frequencies at each locus based on Markov Chain Monte Carlo (MCMC) iterations [51, 52]. Three independent runs were performed and each value for $K$, the number of ancestral populations, ranged from 2 to 10. Each run had 300,000 MCMC iterations, of which the first 200,000 iterations were discarded as burn-in. The optimal value was found to be $K=6$ by comparing runs using the same values of $\mathrm{K}$ from 2 to 10 .

We applied Splits Tree $4.0[53,54]$ and ClonalFrame [35] to assess recombination and mutation frequencies. The split decomposition method was performed based on the concatenated sequences of all alleles of the eight genes. To do ClonalFrame, a total of 100,000 MCMC iterations and three runs were performed, of which the first half was discarded as burn-in. To evaluate the relative impact of mutation rate $(\theta)$ and recombination rate (r) on the whole population of STs, r/ $\theta$ (relative frequency of occurrence of recombination and mutation) and $\mathrm{r} / \mathrm{m}$ (relative impact of recombination and mutation in the diversification of the lineages) were calculated. 


\section{Nucleotide sequence accession numbers}

Allele sequences for the eight genes used in MLST were deposited in the GenBank database under accession numbers from KP033527 to KP034958.

\section{Conclusion}

We developed an MLST scheme for L. plantarum using 186 strains isolated from a range of different sources, and evaluated some basic parameters of its population biology. Our results indicate a low degree of recombination during the evolution of L. plantarum. Remarkably, the sources of isolates were possibly correlated with their diversity and population structure. In future studies we hope to further clarify these results by using more isolates from different sources.

\section{Availability of supporting data}

The sequences data sets supporting the results of this article are available in the GenBank repository of National Center for Biotechnology Information (http:// www.ncbi.nlm.nih.gov/nuccore/), and accession numbers were from KP033527 to KP034958.

\section{Additional files}

Additional file 1: Table S1. Allelic profiles of analyzed L. plantarum strains. Table S2. Bacterial isolates used in this study. Table S3. Information of type strains and reference strains. (DOCX $29 \mathrm{~kb}$ )

Additional file 2: Figure S1. Clonal genealogy inferred from ClonalFrame analysis of our data using a $50 \%$ majority-rule consensus tree from three independent runs. The clades from the ClonalFrame analysis correspond to the shadowed populations identified in Fig. 2 and have therefore been coloured with the same colors as in Fig. 2. (DOC 90 kb)

\section{Abbreviations}

MLST: Multilocus sequence typing; L. plantarum: Lactobacillus plantarum; LAB: Lactic acid bacterium; STs: Sequence types; CCs: Clonal complexes; sSNP: Synonymous single nucleotide polymorphism sites; nSNP: Nonsynonymous single nucleotide polymorphism sit; $\left.\right|_{A}$ : Index of association;

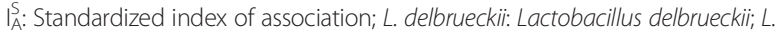
sanfranciscensis: Lactobacillus sanfranciscensis; L. casei: Lactobacillus casei; IMAU: Inner Mongolia Agriculture University; ATCC: American Type Culture Collection; CGMCC: China General Microbiological Culture Collection Center; NCIMB: National Collection of Industrial and Marine Bacteria; $d_{s}$ : Synonymous substitutions; $d_{N}$ : Non-synonymous substitutions; MCMC: Markov Chain Monte Carlo.

\section{Competing interests}

The authors declare no conflicts of interest.

\section{Authors' contributions}

HYX, ZHS, HPZ and BLGMH designed the experiments. HYX, WJL, WYZ, JY, YQS performed the experiments. HYX and ZHS drafted the manuscript. All authors read and approved the final manuscript.

\section{Acknowledgement}

This research was supported by the National Natural Science Foundation of China (Grant No. 31201396), Hi-Tech Research and Development Program of China (863 Planning, Grant No. 2011AA100901 and 2011AA100902), International S\&T Cooperation Program of China (ISTCP, Grant No. 2014DFR31150) and the China Agriculture Research System (Grant No. CARS-37).
Received: 1 July 2015 Accepted: 23 October 2015

Published online: 28 October 2015

\section{References}

1. Siezen RJ, Tzeneva VA, Castioni A, Wels M, Phan HT, Rademaker JL, et al. Phenotypic and genomic diversity of Lactobacillus plantarum strains isolated from various environmental niches. Environ Microbiol. 2010;12(3):758-73.

2. Bringel F, Quénée P, Tailliez P. Polyphasic investigation of the diversity within Lactobacillus plantarum related strains revealed two L. plantarum subgroups. Syst Appl Microbiol. 2001;24(4):561-71.

3. Gardner NJ, Savard T, Obermeier P, Caldwell G, Champagne CP. Selection and characterization of mixed starter cultures for lactic acid fermentation of carrot, cabbage, beet and onion vegetable mixtures. Int J Food Microbiol. 2001;64(3):261-75.

4. Wouters D, Grosu-Tudor S, Zamfir M, De Vuyst L. Applicability of Lactobacillus plantarum IMDO 788 as a starter culture to control vegetable fermentations. J Sci Food Agric. 2013;93(13):3352-61.

5. Zago M, Lanza B, Rossetti L, Muzzalupo I, Carminati D, Giraffa G. Selection of Lactobacillus plantarum strains to use as starters in fermented table olives: Oleuropeinase activity and phage sensitivity. Food Microbiol. 2013;34(1):81-7.

6. Pisano MB, Patrignani F, Cosentino S, Guerzoni ME, Franz CMAP, Holzapfel WH. Diversity and functional properties of Lactobacillus plantarum-group strains isolated from Italian cheese products. Dairy Sci Technol. 2011;91(1):65-76.

7. Zago M, Fornasari ME, Carminati D, Burns P, Suàrez V, Vinderola G, et al. Characterization and probiotic potential of Lactobacillus plantarum strains isolated from cheeses. Food Microbiol. 2011;28(5):1033-40.

8. Rubio R, Aymerich T, Bover-Cid S, Guardia MD, Arnau J, Garriga M. Probiotic strains Lactobacillus plantarum $299 \mathrm{~V}$ and Lactobacillus rhamnosus GG as starter cultures for fermented sausages. Lwt-Food Sci Technol. 2013;54(1):51-6.

9. Zhang H, Liu L, Hao Y, Zhong S, Liu H, Han T, et al. Isolation and partial characterization of a bacteriocin produced by Lactobacillus plantarum BM1 isolated from a traditionally fermented Chinese meat product. Microbiol Immunol. 2013;57(11):746-55.

10. Zhang W, Sun Z, Bilige M, Zhang $H$. Complete genome sequence of probiotic Lactobacillus plantarum P-8 with antibacterial activity. J Biotechnol. 2015;193:41-2.

11. Siezen RJ, van Hylckama VJ. Genomic diversity and versatility of Lactobacillus plantarum, a natural metabolic engineer. Microb Cell Fact. 2011;10.

12. Parente E, Ciocia F, Ricciardi A, Zotta T, Felis GE, Torriani S. Diversity of stress tolerance in Lactobacillus plantarum, Lactobacillus pentosus and Lactobacillus paraplantarum: a multivariate screening study. Int J Food Microbiol. 2010;144(2):270-9.

13. de las Rivas B, Marcobal A, Muñoz R. Development of a multilocus sequence typing method for analysis of Lactobacillus plantarum strains. Microbiology. 2006;152(1):85-93.

14. Huang C-H, Lee F-L, Liou J-S. Rapid discrimination and classification of the Lactobacillus plantarum group based on a partial dnaK sequence and DNA fingerprinting techniques. Antonie Van Leeuwenhoek. 2010;97(3):289-96.

15. López I, Torres C, Ruiz-Larrea F. Genetic typification by pulsed-field gel electrophoresis (PFGE) and randomly amplified polymorphic DNA (RAPD) of wild Lactobacillus plantarum and Oenococcus oeni wine strains. Eur Food Res Technol. 2008;227(2):547-55.

16. Tanganurat W, Quinquis B, Leelawatcharamas V, Bolotin A. Genotypic and phenotypic characterization of Lactobacillus plantarum strains isolated from Thai fermented fruits and vegetables. J Basic Microbiol. 2009;49(4):377-85.

17. Torriani S, Clementi F, Vancanneyt M, Hoste B, Dellaglio F, Kersters K. Differentiation of Lactobacillus plantarum, L. pentosus and L. paraplantarum species by RAPD-PCR and AFLP. Syst Appl Microbiol. 2001;24(4):554-60.

18. Maiden MC, Bygraves JA, Feil E, Morelli G, Russell JE, Urwin R, et al. Multilocus sequence typing: a portable approach to the identification of clones within populations of pathogenic microorganisms. Proc Natl Acad Sci. 1998;95(6):3140-5.

19. Achtman M, Wain J, Weill FX, Nair S, Zhou ZM, Sangal V, et al. Multilocus Sequence Typing as a Replacement for Serotyping in Salmonella enterica. PLoS Pathog. 2012:8(6), e1002776.

20. Ragon M, Wirth T, Hollandt F, Lavenir R, Lecuit M, Le Monnier A, et al. A new perspective on Listeria monocytogenes evolution. PLoS Pathog. 2008;4(9), e1000146. 
21. Oh PL, Benson AK, Peterson DA, Patil PB, Moriyama EN, Roos S, et al. Diversification of the gut symbiont Lactobacillus reuteri as a result of host-driven evolution. The ISME journal. 2010;4(3):377-87.

22. Bachmann H, Starrenburg MJ, Dijkstra A, Molenaar D, Kleerebezem M, Rademaker $J$, et al. Regulatory phenotyping reveals important diversity within the species Lactococcus lactis. Appl Environ Microbiol. 2009;75(17):5687-94.

23. de Las RB, Marcobal A, Munoz R. Allelic diversity and population structure in Oenococcus oeni as determined from sequence analysis of housekeeping genes. Appl Environ Microbiol. 2004;70(12):7210-9.

24. Turner KME, Hanage WP, Fraser C, Connor TR, Spratt BG. Assessing the reliability of eBURST using simulated populations with known ancestry. BMC Microbiol. 2007;7:30.

25. Bao Y, Zhang Y, Li H, Liu Y, Wang S, Dong X, et al. In vitro screen of Lactobacillus plantarum as probiotic bacteria and their fermented characteristics in soymilk. Ann Microbiol. 2012;62(3):1311-20.

26. Jolley KA, Chan MS, Maiden MC. mlstdbNet - distributed multi-locus sequence typing (MLST) databases. Bmc Bioinformatics. 2004;5:86.

27. Tanigawa K, Watanabe K. Multilocus sequence typing reveals a nove subspeciation of Lactobacillus delbrueckii. Microbiol-Sgm. 2011;157:727-38.

28. Kleerebezem M, Boekhorst J, van Kranenburg R, Molenaar D, Kuipers OP Leer $\mathrm{R}$, et al. Complete genome sequence of Lactobacillus plantarum WCFS1. Proc Natl Acad Sci. 2003;100(4):1990-5.

29. Sneath PH, Sokal RR. Numerical taxonomy, The principles and practice of numerical classification. 1973

30. Wang YY, Chen C, Ai LZ, Zhou FF, Zhou ZM, Wang L, et al. Complete genome sequence of the probiotic lactobacillus plantarum ST-III. J Bacteriol. 2011;193(1):313-4.

31. Zhang ZY, Liu C, Zhu YZ, Zhong Y, Zhu YQ, Zheng HJ, et al. Complete Genome Sequence of Lactobacillus plantarum JDM1. J Bacteriol. 2009;191(15):5020-1.

32. Picozzi C, Bonacina G, Vigentini I, Foschino R. Genetic diversity in Italian Lactobacillus sanfranciscensis strains assessed by multilocus sequence typing and pulsed-field gel electrophoresis analyses. Microbiology. 2010;156(7):2035-45.

33. Cai H, Rodríguez BT, Zhang W, Broadbent JR, Steele JL. Genotypic and phenotypic characterization of Lactobacillus casei strains isolated from different ecological niches suggests frequent recombination and niche specificity. Microbiology. 2007;153(8):2655-65.

34. Didelot $X$, Maiden MCJ. Impact of recombination on bacterial evolution. Trends Microbiol. 2010;18(7):315-22.

35. Didelot $X$, Falush D. Inference of bacterial microevolution using multilocus sequence data. Genetics. 2007;175(3):1251-66.

36. Bao Q, Yu J, Liu W, Qing M, Wang W, Chen X, et al. Predominant lactic acid bacteria in traditional fermented yak milk products in the Sichuan Province of China. Dairy Sci Technol. 2012;92(3):309-19.

37. Liu W, Bao Q, Qing M, Chen X, Sun T, Li M, et al. Isolation and identification of lactic acid bacteria from tarag in eastern inner Mongolia of china by $16 \mathrm{~S}$ rRNA sequences and DGGE analysis. Microbiol Res. 2012;167(2):110-5.

38. Yu J, Du X, Wang W, Zhang J, Liu W, Sun Z, et al. Phenotypic and genotypic characteristics of lactic acid bacteria isolated from sour congee in Inner Mongolia of China. J Gen Appl Microbiol. 2011;57(4):197-206.

39. Yu J, Gao W, Qing M, Sun Z, Wang W, Liu W, et al. Identification and characterization of lactic acid bacteria isolated from traditional pickles in Sichuan, China. J Gen Appl Microbiol. 2012;58(3):163-72.

40. Yu J, Sun Z, Liu W, Bao Q, Zhang J, Zhang H. Phylogenetic study of Lactobacillus acidophilus group, L. casei group and L. plantarum group based on partial hsp60, pheS and tuf gene sequences. Eur Food Res Technol. 2012;234(6):927-34.

41. Yu J, Wang W, Menghe B, Jiri M, Wang H, Liu W, et al. Diversity of lactic acid bacteria associated with traditional fermented dairy products in Mongolia. J Dairy Sci. 2011;94(7):3229-41.

42. Zhang J, Liu W, Sun Z, Bao Q, Wang F, Yu J, et al. Diversity of lactic acid bacteria and yeasts in traditional sourdoughs collected from western region in Inner Mongolia of China. Food Control. 2011;22(5):767-74.

43. Bilhere E, Lucas PM, Claisse O, Lonvaud-Funel A. Multilocus sequence typing of Oenococcus oeni: detection of Two subpopulations shaped by intergenic recombination. Appl Environ Microbiol. 2009;75(5):1291-300.

44. Rademaker JLW, Herbet H, Starrenburg MJC, Naser SM, Gevers D, Kelly WJ, et al. Diversity analysis of dairy and nondairy Lactococcus lactis isolates, using a novel multilocus sequence analysis scheme and (GTG)5-PCR fingerprinting. Appl Environ Microbiol. 2007;73(22):7128-37.

45. Tamura K, Stecher G, Peterson D, Filipski A, Kumar S. MEGA6: molecular evolutionary genetics analysis version 6.0. Mol Biol Evol. 2013;30(12):2725-9.

46. Feil EJ, Li BC, Aanensen DM, Hanage WP, Spratt BG. eBURST: Inferring patterns of evolutionary descent among clusters of related bacterial genotypes from multilocus sequence typing data. J Bacteriol. 2004;186(5):1518-30.

47. Jolley KA, Feil EJ, Chan MS, Maiden MC. Sequence type analysis and recombinational tests (START). Bioinform. 2001;17(12):1230-1.

48. Haubold B, Hudson RR. LIAN 3.0: detecting linkage disequilibrium in multilocus data. Linkage Analysis. Bioinform. 2000;16(9):847-8.

49. Rozas J, Sanchez-DelBarrio JC, Messeguer X, Rozas R. DnaSP, DNA polymorphism analyses by the coalescent and other methods. Bioinform. 2003;19(18):2496-7.

50. Falush D, Stephens M, Pritchard JK. Inference of population structure using multilocus genotype data: dominant markers and null alleles. Mol Ecol Notes. 2007;7(4):574-8.

51. Evanno G, Regnaut S, Goudet J. Detecting the number of clusters of individuals using the software STRUCTURE: a simulation study. Mol Ecol. 2005;14(8):2611-20.

52. Pritchard JK, Stephens M, Donnelly P. Inference of population structure using multilocus genotype data. Genetics. 2000;155(2):945-59.

53. Huson DH. SplitsTree: analyzing and visualizing evolutionary data. Bioinform. 1998;14(1):68-73.

54. Huson DH, Bryant D. Application of phylogenetic networks in evolutionary studies. Mol Biol Evol. 2006;23(2):254-67.

\section{Submit your next manuscript to BioMed Central and take full advantage of:}

- Convenient online submission

- Thorough peer review

- No space constraints or color figure charges

- Immediate publication on acceptance

- Inclusion in PubMed, CAS, Scopus and Google Scholar

- Research which is freely available for redistribution 